\title{
Planejamento para a Conservação da Biodiversidade: uma Abordagem Prática e Interdisciplinar de Ensino
}

\author{
Paulo Henrique Ott ${ }^{1} \&$ Juçara Bordin ${ }^{1}$
}

Recebido em 16/10/2020 - Aceito em 28/02/2021 1 Universidade Estadual do Rio Grande do Sul/UERGS. Unidade Hortênsias. São Francisco de Paula/RS, Brasil. < paulo-ott@uergs.edu.br,
jucara-bordin@uergs.edu.br>.

\begin{abstract}
RESUMO - A identificação e proteção de áreas prioritárias para conservação é uma das principais ferramentas utilizadas atualmente para a conservação e uso sustentável dos recursos naturais. A estratégia, amplamente ancorada na Biologia da Conservação, envolve princípios de diferentes áreas do conhecimento, mostrando-se bastante interessante como tema interdisciplinar de ensino. No presente artigo, é apresentada uma proposta de exercício prático de ensino que envolve a análise e priorização de áreas para a conservação da biodiversidade regional. A proposta, além de explorar conceitos fundamentais relacionados à conservação e manejo da fauna e flora brasileira, permite desenvolver importantes habilidades necessárias para a tomada de decisão envolvendo temas socioambientais, os quais muitas vezes podem ser controversos. A atividade foi desenvolvida originalmente como parte de uma disciplina do curso de Mestrado Profissional em Ambiente e Sustentabilidade da Universidade Estadual do Rio Grande do Sul, entre 2016 e 2020, mas a possibilidade de simplificação e adaptação local permite que seja também utilizada como tema gerador de processos educativos ambientais nos níveis fundamental e médio de ensino. Considerando que a extinção de espécies da fauna e da flora regional pode ter um significado concreto para alunos de diferentes regiões do país e que este tema envolve um conteúdo problematizador, a adaptação desse exercício para diferentes realidades locais pode permitir reflexões mais amplas sobre o uso sustentável dos recursos naturais e a conservação da biodiversidade brasileira. Desta forma, o presente artigo busca estimular a difusão e o debate desse tema em diferentes níveis de ensino, visando o desenvolvimento do senso crítico e formação de cidadãos socioambientalmente responsáveis e que compreendam a importância e os desafios da implementação de áreas protegidas.
\end{abstract}

Palavras-chave: Área protegida; educação ambiental; espécie ameaçada; seleção de reservas.

\section{Planning for Biodiversity Conservation: a Practical and Interdisciplinary Teaching Approach}

ABSTRACT - The identification and protection of priority areas for conservation is one of the main tools currently applied for protection and sustainable use of natural resources. The strategy, widely anchored in Conservation Biology, involves principles from different areas of knowledge, proving to be quite interesting as an interdisciplinary teaching theme. In this article, we present a proposal for a practical teaching exercise that involves the analysis and prioritization of areas for the conservation of regional biodiversity. The proposal explores important scientific contents related to the conservation and management of the Brazilian fauna and flora. Moreover, it allows the development of important skills necessary for decision-making related to socio-environmental issues, which can often be controversial. The activity was originally developed as part of a professional master's course (Mestrado Profissional em Ambiente e Sustentabilidade da Universidade Estadual do Rio Grande do Sul), between 2016 and 2020, but the possibility of simplification and local adaptation allows it to also be used as a "generative theme" of environmental educational processes at primary and secondary levels of education. Considering that the extinction of species of regional fauna and flora may have a concrete meaning for students from different regions of the country and that this theme involves a problematizing issue, the adaptation of this exercise to different local realities may allow a broader reflection on the sustainable use of natural resources and conservation of the Brazilian biodiversity. In this way, this article seeks to stimulate the dissemination and debate of this theme at different teaching 
levels, aiming at the development of critical sense and the formation of socially and environmentally responsible citizens who understand the importance and challenges of implementing protected areas.

Keywords: Protected area; environmental education; endangered species; reserve selection.

\title{
Planificación para la Conservación de la Biodiversidad: un Enfoque de Enseñanza Práctico y Interdisciplinario
}

\begin{abstract}
RESUMEN - La identificación y protección de áreas prioritarias para la conservación es una de las principales herramientas que se utilizan actualmente para la conservación y uso sostenible de los recursos naturales. La estrategia, ampliamente anclada en la Biología de la Conservación, involucra principios de diferentes áreas del conocimiento, resultando bastante interesante como tema didáctico interdisciplinario. En este artículo presentamos una propuesta de ejercicio práctico docente que involucra el análisis y priorización de áreas para la conservación de la biodiversidad regional. La propuesta, además de explorar conceptos fundamentales relacionados con la conservación y manejo de la fauna y la flora brasileña, permite el desarrollo de importantes habilidades necesarias para la toma de decisiones que involucran temas socioambientales, que a menudo pueden ser controvertidos. La actividad se desarrolló originalmente como parte de una disciplina de una maestría profesional (Mestrado Profissional em Ambiente e Sustentabilidade da Universidade Estadual do Rio Grande do Sul), entre 2016 y 2020, pero la posibilidad de simplificación y adecuación local permite que también sea utilizada como un tema que genera procesos educativos ambientales en los niveles elemental y secundario de las escuelas. Considerando que la extinción de especies de fauna y flora regional puede tener un significado concreto para estudiantes de diferentes regiones del país y que esta temática involucra un contenido problematizador, la adecuación de este ejercicio a diferentes realidades locales puede permitir reflexiones más amplias sobre el uso sostenible de los recursos naturales y la conservación de la biodiversidad brasileña. De esta manera, este artículo busca estimular la difusión y el debate de esta temática en los diferentes niveles de la educación, apuntando al desarrollo del sentido crítico y la formación de ciudadanos social y ambientalmente responsables que comprendan la importancia y desafíos de implementar áreas protegidas.
\end{abstract}

Palabras clave: Área protegida; educación ambiental; especie amenazada; selección de reservas.

\section{Introdução}

A conservação da biodiversidade e uso sustentável dos recursos naturais é um dos grandes desafios de nossa sociedade. A velocidade $e$ a magnitude com que estamos explorando $e$ modificando os ecossistemas naturais são sem precedentes em nossa história (Pimm et al., 2014). Como resultado, a taxa de extinção de espécies na atualidade é comparável às taxas das grandes extinções em massa, conhecidas pelos registros fósseis, que ocorreram ao longo da evolução de nosso planeta (Fauth, 2002; Barnosky et al., 2011).

Diante desse cenário, existe a premente necessidade de encontrar estratégias que possam garantir a conservação das espécies e o funcionamento dos ecossistemas em longo prazo. Dentre as diferentes estratégias existentes, têm se destacado as iniciativas que visam à priorização dos alvos a serem conservados. Nesse sentido, a identificação e proteção de áreas prioritárias tem sido um dos grandes pilares para a conservação da biodiversidade e uso sustentável dos recursos naturais no mundo todo (Margules et al., 2002; Olson \& Dinerstein, 2002; Eken et al., 2004; MMA, 2007; D'Arrigo et al., 2020).

O princípio por trás dessa estratégia seria que, na impossibilidade de se conservar todos os ecossistemas do mundo, ou mesmo os diferentes ambientes presentes em um território, essas áreas prioritárias garantiriam a conservação de uma parcela significativa da biodiversidade existente. No clássico artigo de Myers et al. (2000), os autores identificaram 25 regiões no mundo que, embora representassem apenas $1,4 \%$ da superfície do planeta, compreenderiam cerca de $44 \%$ das espécies de plantas vasculares e $35 \%$ de todas as espécies de vertebrados terrestres existentes. Estas regiões, chamadas de hotspots, deveriam, portanto, ser priorizadas tanto em relação aos investimentos quanto às ações para a conservação da biodiversidade.

Neste contexto, a criação de áreas protegidas tem sido uma importante ferramenta 
para a conservação da biodiversidade (Brooks et al., 2004; Watson et al., 2014). O primeiro parque nacional criado no mundo nos moldes que conhecemos atualmente foi $\mathrm{O}$ Parque Yellowstone, nos Estados Unidos, em 1872. Esta área foi estabelecida na época principalmente em função de seus atributos cênicos e potencial para atividades de lazer (Phillips, 2004). Desde então, inúmeras áreas protegidas têm sido criadas no mundo todo, com diferentes objetivos, dimensões, tipos de governança e formas de manejo (Dudley et al., 2010; Watson et al., 2014). No Brasil, os primeiros parques nacionais foram criados no final da década de 1930, incluindo Itatiaia, em 1937, e Iguaçu e Serra dos Órgãos em 1939 (Mittermeier et al., 2005), embora outros modelos de áreas protegidas, como a Reserva Florestal Estadual de São Paulo, tenham sido estabelecidos ainda no século XX (Franco et al., 2015).

Atualmente, segundo o Painel Dinâmico de Informações sobre as unidades de conservação (UCs) no Brasil, existem 334 UCs federais no país (ICMBio, 2021), além das estaduais e municipais. As UCs federais estão classificadas em doze categorias, conforme o Sistema Nacional de Unidades de Conservação da Natureza (SNUC) (Brasil, 2002). Essas distintas categorias estão reunidas em dois grandes grupos, de acordo com seus objetivos de manejo e possibilidades de utilização de seus recursos: i) UCs de proteção integral, as quais têm como objetivo principal preservar a natureza, sendo admitido apenas o uso indireto dos seus recursos naturais, com exceção dos casos específicos previstos no SNUC; e ii) UCs de uso sustentável, nas quais é permitido o uso sustentável dos recursos naturais existentes em consonância com a conservação da biodiversidade (Brasil, 2002). Além dessa diferença básica entre os dois grandes grupos, cada uma das categorias previstas no SNUC apresenta objetivos específicos e certas particularidades, relacionadas, por exemplo, à possibilidade de visitação pública e à ocupação humana (Tabela 1).

Apesar do aumento expressivo do número de UCs criadas nas últimas décadas em todo o mundo, a perda da biodiversidade e a extinção de espécies são problemas crescentes. Segundo a União Internacional para a Conservação da Natureza (UICN - em inglês, IUCN), mais de 32.000 espécies estão globalmente ameaçadas de extinção (IUCN, 2020). Além disso, alguns estudos têm demonstrado que cerca de $1 / 5$ dos vertebrados terrestres, além de espécies de outros grupos, não estão resguardados por nenhuma área protegida (Rodrigues et al., 2004; Venter et al., 2014).

Com o intuito de ampliar as ações para a conservação da biodiversidade em âmbito mundial, durante a $10^{\text {a }}$ Conferência das Partes da Convenção sobre Diversidade Biológica (COP-10), realizada em 2010 na cidade de Nagoya, Província de Aichi, Japão, foi aprovado o Plano Estratégico de Biodiversidade para o período de 2011 a 2020. O documento, que ficou conhecido como "Metas de Aichi", propôs aos países signatários, incluindo o Brasil, o compromisso de implantar cinco objetivos estratégicos para a conservação da biodiversidade, com base em 20 metas definidas. A Meta 11 desse documento estabelece que, até 2020, pelo menos $17 \%$ de áreas terrestres e de águas continentais e $10 \%$ de áreas marinhas e costeiras do planeta seriam preservadas por meio de áreas protegidas (CDB, 2011; Prates \& Irving, 2015).

Contudo, além dos acordos internacionais e dos instrumentos legais para criação das áreas protegidas, é importante também estabelecer critérios para a escolha das áreas a serem preservadas (Pinto \& Grelle, 2009). A definição desses critérios, bem como das ações de manejo propostas, são questões centrais da Biologia da Conservação. Historicamente, muitas áreas protegidas foram criadas em virtude da existência de atributos cênicos interessantes para a visitação pública, da presença de espécies carismáticas, ou mesmo da facilidade para aquisição das áreas. Posteriormente, outros objetivos passaram a direcionar a criação destas áreas, incluindo a conservação da biodiversidade e a proteção de serviços ecossistêmicos (Watson et al., 2014; Franco et al., 2015).

Mais recentemente, grandes áreas protegidas também têm sido criadas, especialmente em regiões marinhas longínquas da costa, com o principal intuito de cumprir com as metas internacionais estabelecidas, prevalecendo assim critérios políticos sobre ecológicos (Jones \& De Santo, 2016; Silva, 2019). Embora essas áreas possam também conter um importante conjunto de espécies e ecossistemas, a utilização de critérios ecológicos para estas escolhas pode aumentar de forma significativa a efetividade para a conservação da biodiversidade (Magris \& Pressey, 2018; Visconti et al., 2019). 
Tabela 1 - Categorias das unidades de conservação (UCs), segundo o Sistema Nacional de Unidades de Conservação da Natureza (SNUC).

\begin{tabular}{|c|c|c|c|c|}
\hline & Categoria & Objetivos de criação & Visitação pública & Ocupação humana \\
\hline \multirow{5}{*}{ 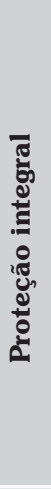 } & Estação ecológica & Preservação da natureza e realização de pesquisas científicas & Não* & Não \\
\hline & Reserva biológica & $\begin{array}{l}\text { Preservação integral da biota e demais atributos naturais } \\
\text { existentes em seus limites }\end{array}$ & Não* & Não \\
\hline & Parque nacional & $\begin{array}{l}\text { Preservação de ecossistemas naturais de grande relevância } \\
\text { ecológica e beleza cênica }\end{array}$ & Sim** & Não \\
\hline & Monumento natural & $\begin{array}{l}\text { Preservação de sítios naturais raros, singulares ou de grande } \\
\text { beleza cênica }\end{array}$ & $\operatorname{Sim} * *$ & Sim \\
\hline & $\begin{array}{l}\text { Refúgio de vida } \\
\text { silvestre }\end{array}$ & $\begin{array}{l}\text { Proteção de ambientes naturais onde se asseguram condições } \\
\text { para a existência ou reprodução de espécies ou comunidades } \\
\text { da flora local e da fauna residente ou migratória }\end{array}$ & Sim** & Sim \\
\hline \multirow{7}{*}{ 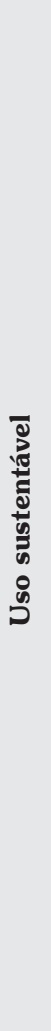 } & $\begin{array}{l}\text { Área de proteção } \\
\text { ambiental }\end{array}$ & $\begin{array}{l}\text { Proteção da diversidade biológica, disciplinando o processo } \\
\text { de ocupação de forma a assegurar a sustentabilidade do uso } \\
\text { dos recursos naturais }\end{array}$ & Sim** & Sim \\
\hline & $\begin{array}{l}\text { Área de relevante } \\
\text { interesse ecológico }\end{array}$ & $\begin{array}{l}\text { Manutenção dos ecossistemas naturais de importância } \\
\text { regional ou local, regulando o uso dessas áreas, de modo a } \\
\text { compatibilizá-lo com os objetivos de conservação da natureza }\end{array}$ & Sim** & Pouca ou nenhuma \\
\hline & Floresta nacional & $\begin{array}{l}\text { Uso sustentável dos recursos florestais e desenvolvimento de } \\
\text { pesquisa científica, com ênfase em métodos para exploração } \\
\text { sustentável de florestas nativas }\end{array}$ & Sim** & Sim \\
\hline & Reserva extrativista & $\begin{array}{l}\text { Área utilizada por populações extrativistas tradicionais, } \\
\text { tendo como objetivos básicos proteger os meios de vida e a } \\
\text { cultura dessas populações, assegurando o uso sustentável dos } \\
\text { recursos naturais }\end{array}$ & Sim** & $\begin{array}{l}\text { Populações } \\
\text { extrativistas } \\
\text { tradicionais }\end{array}$ \\
\hline & Reserva de fauna & $\begin{array}{l}\text { Área natural com populações animais de espécies nativas } \\
\text { adequada para estudos técnico-científicos sobre o manejo } \\
\text { econômico sustentável de recursos faunísticos }\end{array}$ & $\operatorname{Sim}^{* *}$ & Não \\
\hline & $\begin{array}{l}\text { Reserva de } \\
\text { desenvolvimento } \\
\text { sustentável }\end{array}$ & $\begin{array}{l}\text { Área natural que abriga populações tradicionais, cuja } \\
\text { existência baseia-se em sistemas sustentáveis de exploração } \\
\text { dos recursos naturais. Tem por objetivo a preservação da } \\
\text { natureza associada à manutenção e melhoria dos modos de } \\
\text { exploração dos recursos naturais e da qualidade de vida das } \\
\text { populações tradicionais }\end{array}$ & $\operatorname{Sim}^{* *}$ & $\begin{array}{l}\text { Populações } \\
\text { tradicionais }\end{array}$ \\
\hline & $\begin{array}{l}\text { Reserva particular do } \\
\text { patrimônio natural }\end{array}$ & $\begin{array}{l}\text { Área privada, criada por iniciativa do proprietário, } \\
\text { estabelecida com o objetivo de conservar a diversidade } \\
\text { biológica }\end{array}$ & $\operatorname{Sim}^{* *}$ & $\begin{array}{l}\text { Proprietário e } \\
\text { funcionários da UC }\end{array}$ \\
\hline
\end{tabular}

*É proibida a visitação pública, exceto quando com objetivo educacional e de acordo com o disposto no plano de manejo da UC.

**A visitação pública é permitida, desde que compatível com os interesses locais e de acordo com o disposto no plano de manejo da UC.

Dentre os critérios propostos para a escolha de áreas prioritárias para a conservação estão, por exemplo, a riqueza de espécies, a presença de espécies ameaçadas ou raras, bem como a fragilidade ambiental (Eken et al., 2004; Pressey et al., 2007; D’Arrigo et al., 2020). Além disso, outros aspectos relacionados à ecologia de paisagem como tamanho, distância e formato das áreas, bem como a proximidade com áreas alteradas ou com populações humanas, também podem influenciar o sucesso e efetividade das mesmas (Donaldson et al., 2017). No entanto, a escolha e manejo de áreas protegidas é um tema que envolve não apenas aspectos ecológicos, como também aspectos econômicos e diferentes visões e percepções a respeito da participação da sociedade em relação à gestão e repartição dos recursos naturais (Ferreira, 2004; Vivacqua \& Vieira, 2005).

Em função dessa interdisciplinaridade, o tema se mostra bastante interessante como facilitador e integrador de vários conceitos de Ecologia 
e Biologia da Conservação. Além disso, nas últimas décadas, tem sido enfatizada a necessidade de desenvolver habilidades no âmbito escolar $e$ acadêmico para a tomada de decisão relacionada à sustentabilidade ambiental (Santos \& Mortimer, 2001; Emery et al., 2015; Dionor et al., 2020). Em virtude da magnitude do impacto da humanidade sobre os recursos naturais atuais, alguns autores inclusive destacam que o ensino de Ecologia $e$ Biologia da Conservação deve ser considerado um dos pilares da boa cidadania em qualquer nação (Orr, 1992; Trombulak et al., 2004).

No Brasil, as questões ligadas à conservação e preservação da natureza estão inseridas nas competências definidas pela Base Nacional Comum Curricular (BNCC), a qual corresponde a um documento sintetizado pelo Conselho Nacional de Educação que estabelece o conjunto de aprendizagens básicas essenciais e indispensáveis a que todos os estudantes têm direito (Brasil, 2017). Conforme a BNCC, entre as competências básicas que devem ser desenvolvidas nos estudantes, ainda durante o Ensino Fundamental, estão o desenvolvimento da capacidade de argumentar, formular e defender ideias e pontos de vista e tomar decisões que respeitem e promovam os direitos humanos, a consciência socioambiental e o consumo responsável, visando o cuidado de si mesmo, dos outros e do planeta.

O próprio Instituto Chico Mendes de Conservação da Biodiversidade (ICMBio), responsável pela gestão das UCs federais, estabeleceu, em 2010, em conjunto com o Ministério do Meio Ambiente, a Estratégia Nacional de Comunicação e Educação Ambiental (ENCEA) a ser implementada no âmbito do SNUC (Brasil, 2010). Este documento aponta diversos princípios e diretrizes relacionados à divulgação e valorização das UCs. Dentre as diretrizes existentes, está o estímulo à inserção das UCs como temática nos processos educativos formais e não-formais, destacando-se a importância da produção de materiais de ensino-aprendizagem.

Diante disso, no presente artigo é apresentado um exercício prático interdisciplinar de ensino relacionado à seleção de áreas prioritárias para conservação. A ideia central do artigo é estimular a difusão e debate deste tema em diferentes níveis de ensino, visando o desenvolvimento do senso crítico $e$ a formação de cidadãos socioambientalmente responsáveis que compreendam a importância e os desafios da implementação de áreas protegidas.

\section{Metodologia}

A atividade foi desenvolvida originalmente entre os anos de 2016 e 2020, como parte da disciplina de Conservação e Manejo da Biodiversidade do Curso de Mestrado Profissional em Ambiente e Sustentabilidade da Universidade Estadual do Rio Grande do Sul, o qual é composto por três linhas de pesquisa: i) Conservação e Manejo da Biodiversidade; ii) Sociedade, Ambiente e Desenvolvimento; e iii) Tecnologias Sustentáveis para o desenvolvimento.

A base conceitual do exercício foi concebida a partir das ideias apresentadas no diagrama do artigo científico Planning for Biodiversity publicado na revista Science (Pimm \& Lawton, 1998). Neste artigo, os autores apresentam uma situação hipotética na qual diferentes estratégias poderiam ser adotadas para preservar um conjunto de espécies (representadas simplesmente por letras), incluindo espécies raras.

A lógica do exercício proposto é bastante semelhante, mas incorpora uma situação mais realista, na qual são utilizadas espécies reais regionais sobre as quais os próprios alunos buscam diversas informações, a partir das quais farão suas escolhas e tomarão suas decisões. A paisagem proposta está representada em um diagrama composto por 64 células, ficticiamente com áreas de 5 x $5 \mathrm{~km}$ cada, que contêm um conjunto de 13 espécies da fauna e 13 espécies da flora regional, no caso do estado do Rio Grande do Sul (RS). Por simplificação, as espécies são apresentadas apenas com códigos de letras (de "A" à "Z"). O diagrama está organizado em oito colunas, representadas por letras (a-h), e oito linhas, indicadas por números (1-8). Para facilitar a discussão dos resultados, as células são identificadas pela combinação de um número e uma letra que representam sua posição no diagrama. Assim, a décima célula, por exemplo, pode ser indicada pelo código " $2 \mathrm{~b}$ " (Figura 1). Em adição ao diagrama com a distribuição das espécies, é apresentada uma tabela com os nomes científicos e comuns das espécies correspondentes aos códigos (Tabela 2). Como elementos adicionais na paisagem, foram ainda acrescentados $e$ sinalizados no diagrama uma Área de Proteção Ambiental (APA) municipal, uma nascente e um rio com $20 \mathrm{~m}$ de largura. Estes últimos elementos foram inseridos para possibilitar uma discussão a respeito das Áreas de Preservação Permanente previstas no Código Florestal Brasileiro (Brasil, 2012). 
As espécies ocorrentes foram selecionadas a priori pelos professores, com base no conhecimento sobre a biodiversidade na região onde a disciplina estava sendo ofertada (Campos de Cima da Serra, Nordeste do RS), a fim de tornar a experiência mais próxima da realidade dos alunos (Tabela 2). Procurou-se selecionar espécies pertencentes a diferentes grupos taxonômicos, bem como espécies com diferentes padrões de distribuição geográfica e em diferentes estados de conservação. A seleção das espécies em diferentes estados de conservação foi realizada a partir da análise das listas oficiais de espécies da fauna e flora ameaçadas de extinção no estado (Rio Grande do Sul, 2014a, 2014b). Em adição às espécies nativas, foram também incluídas na paisagem espécies exóticas invasoras, constantes na Portaria SEMA $n^{\circ} 79$ de 31 de outubro de 2013, publicada pelo governo do estado (Rio Grande do Sul, 2013).

\begin{tabular}{|c|c|c|c|c|c|c|c|c|}
\hline & $\mathbf{a}$ & b & c & d & e & f & $\mathbf{g}$ & h \\
\hline \multirow{2}{*}{1} & AGI & AGI & $\mathrm{HI}$ & $\mathrm{HI}$ & $\mathrm{CM}$ & $\mathrm{ACM}$ & AEHM & AEHM \\
\hline & $\mathrm{RT}$ & RT & ORW & OSTW & RW & RW & STW & STW \\
\hline \multirow{2}{*}{2} & $\mathrm{ABHI}$ & AHIM & $\mathrm{BDH}$ & $\mathrm{BDH}$ & BDFIM & BDFIM & ACEJM & ACE \\
\hline & $\mathrm{RT}$ & ORV & ORTW & STW & OSUW & OSUW & TWZ & QWZ \\
\hline \multirow{2}{*}{3} & $\mathrm{ABHI}$ & $\mathrm{AHI}$ & $\mathrm{BH}$ & $\mathrm{BDH}$ & $\mathrm{BDF}$ & $\mathrm{BDF}$ & BDF & $\mathrm{BDF}$ \\
\hline & $\mathrm{RX}$ & ORVX & $\mathrm{RX}$ & RSX & OSU & OSU & QSU & QS \\
\hline \multirow{2}{*}{4} & BEIK & EGK & ABDEG & ABDG & DFGH & AHM & ADFI & ADFI \\
\hline & $\mathrm{RT}$ & PRX & PR & $\mathrm{RT}$ & NTX & TVW & STV & SV \\
\hline \multirow{2}{*}{5} & CEGI & $\mathrm{ACE}$ & $\mathrm{BCG}$ & BG & ACGL & ABIL & ABEIL & ABEIL \\
\hline & RTWZ & $\mathrm{TZ}$ & PRTY & RTWY & TWZ & TWZ & WZ & RW \\
\hline \multirow{2}{*}{6} & $\mathrm{EGH}$ & AHKM & ABG & BG & $\mathrm{BCH}$ & $\mathrm{BHI}$ & $\mathrm{BHI}$ & $\mathrm{BHI}$ \\
\hline & TWZ & WZ & OPT & TXY & WXZ & RT & OX & PR \\
\hline \multirow{2}{*}{7} & AG & ACKM & CGKM & CGK & CGHK & $\mathrm{CH}$ & $\mathrm{BD}$ & $\mathrm{BD}$ \\
\hline & TWZ & $\mathrm{W}$ & W & TW & WXZ & ORWX & OPR & OPR \\
\hline \multirow{2}{*}{8} & $\mathrm{AC}$ & AK & ACK & $A C$ & $\mathrm{AC}$ & AC & $\mathrm{ACH}$ & $\mathrm{AH}$ \\
\hline & TWZ & TW & W & TW & RTW & ORW & $\mathrm{R}$ & OPR \\
\hline
\end{tabular}

Figura 1 - Diagrama da distribuição da fauna e flora regional utilizado no exercício prático proposto, incluindo espécies nativas e exóticas. As células em verde claro ("1c" e "1d") representam uma Área de Proteção Ambiental Municipal, onde está localizada a nascente que abastece o rio da região, representado em azul. Fonte: Autores. 
Tabela 2 - Lista das espécies da flora e fauna do Rio Grande do Sul, incluindo nativas e exóticas, utilizadas no exercício prático proposto.

\begin{tabular}{|c|c|c|}
\hline Código & Espécie & Nome Comum \\
\hline A & Achyrocline satureioides (Lam.) DC & Marcela \\
\hline B & Araucaria angustifolia (Bert.) O. Kuntze & Araucária \\
\hline C & Baccharis crispa Spreng. & Carqueja \\
\hline $\mathrm{D}$ & Bryopteris diffusa (Sw.) Nees & Musgo \\
\hline E & Butia eriospatha (Mart.) Becc. & Butiá \\
\hline $\mathrm{F}$ & Drimys brasiliensis Miers & Casca-de-anta \\
\hline G & Eugenia involucrata DC. & Cerejeira-do-mato \\
\hline $\mathrm{H}$ & Ilex paraguariensis A. St.-Hil. & Erva-mate \\
\hline I & Microgramma squamulosa (Kaulf.) de la Sota & Samambaia \\
\hline J & Parodia rechensis (Buining) F.H. Brandt & Cactos \\
\hline K & Pinus elliottii Engelm. & Pinus \\
\hline $\mathrm{L}$ & Trichocline macrocephala Less. & Cravo-do-campo-vermelho \\
\hline M & Ulex europaeus L. & Tojo \\
\hline $\mathrm{N}$ & Aburria jacutinga (Spix, 1825) & Jacutinga \\
\hline $\mathrm{O}$ & Alouatta guariba clamitans Cabrera, 1940 & Bugio-ruivo \\
\hline $\mathrm{P}$ & Amazona vinacea (Kuhl, 1820) & Papagaio-de-peito-roxo \\
\hline $\mathrm{Q}$ & Bothrops jararacussu Lacerda, 1884 & Jararacuçu \\
\hline $\mathrm{R}$ & Cyanocorax caeruleus (Vieillot, 1818) & Gralha-azul \\
\hline S & Epactionotus itaimbezinho Reis \& Schaefer, 1998 & Cascudinho \\
\hline $\mathrm{T}$ & Galictis cuja (Molina, 1782) & Furão \\
\hline U & Hylodes meridionalis (Mertens, 1927) & Rã-da-cachoeira \\
\hline $\mathrm{V}$ & Lithobates catesbeianus (Shaw, 1802) & Rã-touro \\
\hline $\mathrm{W}$ & Lycalopex gymnocercus (G. Fischer, 1814) & Graxaim \\
\hline $\mathrm{x}$ & Melipona bicolor schencki Gribodo, 1893 & Abelha-guaraipo \\
\hline Y & Puma concolor (Linnaeus, 1771) & Leão-Baio; Puma \\
\hline Z & Xanthopsar flavus (Gmelin, 1788) & Veste-amarela \\
\hline
\end{tabular}

Passo 1 -A primeira atividade a ser realizada pelos alunos envolve a avaliação das espécies com base em três critérios de priorização: i) estado de conservação; ii) endemismo (i.e. amplitude da distribuição); e iii) raridade, aqui definida como percentagem da área de ocorrência da espécie em relação à paisagem analisada.

Para cada um desses critérios, foi utilizado um sistema de pontuação similar à metodologia aplicada em um estudo de priorização de plantas medicinais no Himalaia (Tali et al., 2019), sendo os valores mais altos atribuídos para aquelas espécies com maior prioridade de conservação (Tabela 3). Neste sentido, por exemplo, foi atribuída uma pontuação mais alta a uma espécie considerada "Criticamente em Perigo" (CR) do que uma espécie "Vulnerável" (VU), visto a probabilidade de extinção da primeira ser mais elevada (IUCN, 
2012; Figura 2). No caso das espécies exóticas, por outro lado, foram atribuídos valores nulos ou negativos em relação aos critérios avaliados para destacar que a introdução de espécies exóticas está entre as principais ameaças à conservação da biodiversidade na atualidade (Ziller \& Zalba, 2007; IUCN, 2020).

Tabela 3 - Valores atribuídos a cada um dos critérios avaliados para priorização das espécies da fauna e flora em um contexto regional.

\begin{tabular}{|c|c|c|c|c|c|}
\hline $\begin{array}{c}\text { Critério } 1 \\
\text { (Estado de conservação) }\end{array}$ & Valor & $\begin{array}{c}\text { Critério } 2 \\
\text { (Endemismo) }\end{array}$ & Valor & $\begin{array}{l}\text { Critério } 3 \\
\text { (Raridade) }\end{array}$ & Valor \\
\hline Criticamente em Perigo (CR) & 5 & Rio Grande do Sul & 5 & $\begin{array}{l}<10 \% \text { da paisagem ( } 1 \text { a } 6 \\
\text { células) }\end{array}$ & 5 \\
\hline Em Perigo (EN) & 4 & Brasil & 4 & $\begin{array}{l}10-25 \% \text { da paisagem ( } 7 \text { a } 16 \\
\text { células) }\end{array}$ & 4 \\
\hline Vulnerável (VU) & 3 & América do Sul & 3 & $\begin{array}{l}25-50 \% \text { da paisagem (17 a } 32 \\
\text { células) }\end{array}$ & 3 \\
\hline $\begin{array}{l}\text { Quase Ameaçado (NT); Dados } \\
\text { Deficientes (DD) }\end{array}$ & 2 & Américas & 2 & $\begin{array}{l}50-75 \% \text { da paisagem (33 a } 48 \\
\text { células) }\end{array}$ & 2 \\
\hline $\begin{array}{l}\text { Pouco Preocupante (LC) e } \\
\text { Não Avaliado (NE) }\end{array}$ & 1 & Cosmopolita & 1 & $\begin{array}{l}>75 \% \text { da paisagem (>48 } \\
\text { células) }\end{array}$ & 1 \\
\hline $\begin{array}{l}\text { Não Aplicável (NA) (Espécies } \\
\text { Exóticas)* }\end{array}$ & 0 & Exótica & -1 & $\begin{array}{l}\text { Exóticas (independente da } \\
\text { frequência de ocorrência) }\end{array}$ & 0 \\
\hline
\end{tabular}

* Segundo as diretrizes para uso das categorias da lista vermelha de espécies ameaçadas em nível regional e nacional, as espécies exóticas não deveriam ser avaliadas em relação ao seu grau de conservação, devendo ser atribuída a categoria "Não Aplicável” (NA) (IUCN, 2012).
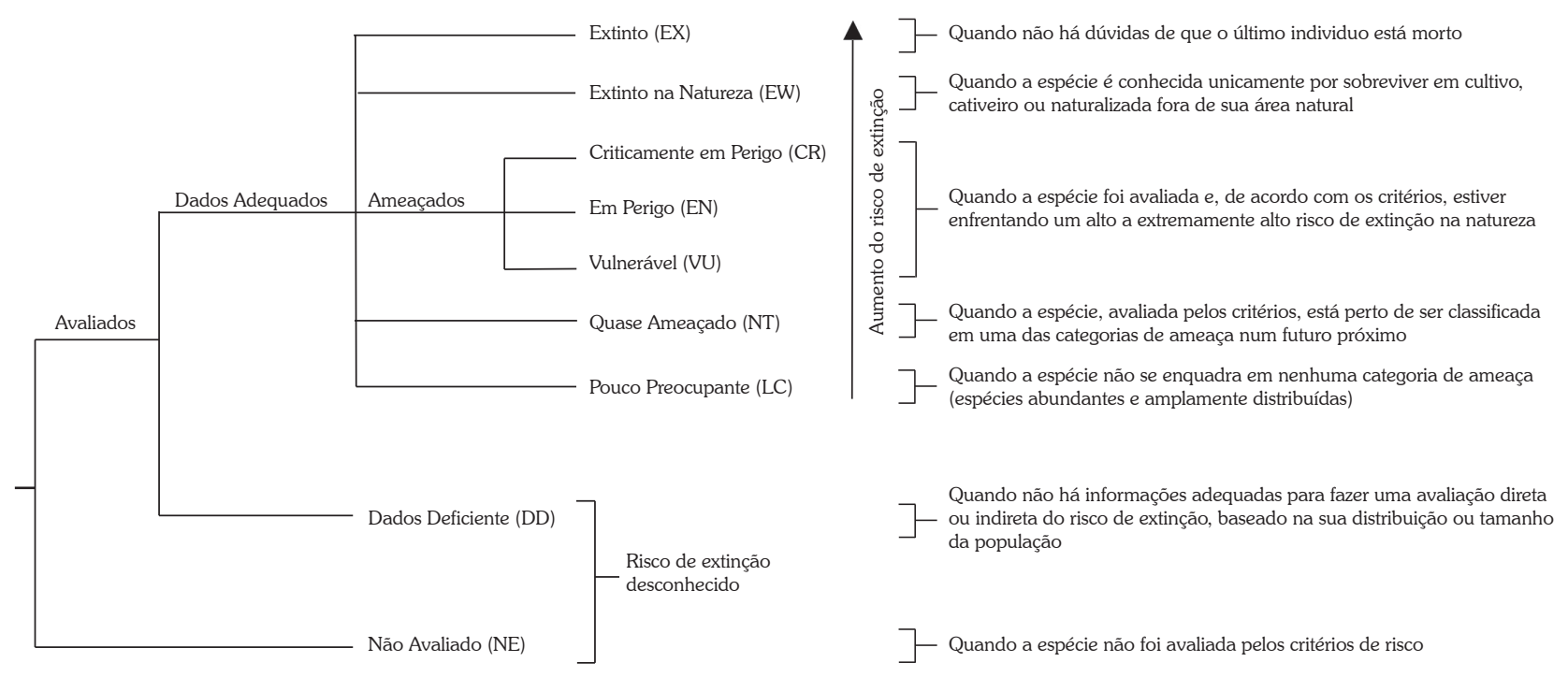

Figura 2 - Representação das relações das diferentes categorias de ameaça da UICN utilizadas em nível regional (IUCN, 2012). Fonte: Autores, adaptado de Rodrigues et al. (2006). 
Passo 2 - Para a avaliação de cada uma das espécies em relação aos três critérios de priorização estabelecidos, é proposto que os alunos pesquisem as informações nas seguintes fontes de dados:

\section{Estado de conservação:}

a. Lista das espécies da fauna ameaçadas do RS (Decreto Estadual n 51.797, 2014a);

b. Lista das espécies da flora ameaçadas do RS (Decreto Estadual n 52.109, 2014b).

Observação: para aqueles estados brasileiros que não possuem listas oficiais de espécies ameaçadas, podem ser utilizadas tanto as Listas Brasileiras de Fauna e Flora Ameaçadas (ICMBio, 2018; Martinelli \& Moraes, 2013) ou a avaliação global da UICN (IUCN, 2020).

\section{Endemismo:}

a. Fauna - Mapas de distribuição geográfica das espécies disponíveis na página eletrônica da UICN (https://www. iucnredlist.org);

b. Flora - Página eletrônica da Flora do Brasil 2020 (http://floradobrasil.jbrj.gov. br/).

Observação: Para a confirmação se uma espécie da fauna ou flora é nativa ou exótica, podem ainda ser utilizados os bancos de dados do Instituto Hórus de Desenvolvimento e Conservação Ambiental (http://bd.institutohorus.org.br/www/) ou da UICN (https://www.iucn.org/node/23891).

\section{Raridade:}

a. Informação obtida diretamente no diagrama (Figura 1), a partir do cálculo do percentual de células com a presença das espécies.

Uma vez que a pontuação máxima estabelecida para cada um dos critérios é igual a 5 , o valor conservativo máximo atribuído a uma espécie será igual a 15 pontos.

Passo 3 - Com base na distribuição das espécies nas diferentes unidades territoriais da paisagem (i.e. 64 células), deve ser calculado o valor conservativo para cada célula, a partir da soma dos valores individuais de cada uma das espécies presentes. Por exemplo, a célula " $3 c$ " (19 célula) contendo duas espécies da flora
( $\mathrm{B}=9$ pontos; $\mathrm{H}=7$ pontos) e duas da fauna ( $\mathrm{R}=7$ pontos; $\mathrm{X}=11$ pontos), com base nos critérios definidos na Tabela 3, possui um valor conservativo igual a 34 pontos.

Passo 4 - A partir de todas as informações levantadas sobre a paisagem e as espécies presentes, os alunos, em grupos de quatro ou cinco integrantes, são provocados a discutir e apresentar aos demais grupos as seguintes situações de manejo:

a. Indicação de quatro áreas prioritárias (i.e. quatro células) para a conservação da fauna e quatro áreas para a conservação da flora regional, justificando os critérios utilizados;

b. Indicação de quatro áreas prioritárias gerais (i.e. quatro células) para a conservação da biodiversidade, considerando concomitantemente as espécies da fauna e da flora, justificando os critérios utilizados;

c. Diante das quatro áreas prioritárias gerais indicadas, que tipos de UCs poderiam ser criadas? A escolha da categoria sugerida deve ser justificada, incluindo reflexões sobre a possibilidade ou não da realização de atividades de uso sustentável nas áreas selecionadas;

d. Supondo que houvesse a demanda para a definição de uma zona industrial de $75 \mathrm{~km}^{2}$ (i.e. três células) na área de estudo, qual área seria proposta no intuito de minimizar o impacto ambiental? Cada grupo deve delimitar a área (células) onde esta zona seria estabelecida, indicando ainda quais medidas seriam adotadas para minimizar os seus potenciais impactos ambientais.

Nesta etapa do trabalho, é importante o professor lembrar que a escolha das quatro áreas prioritárias de cada grupo deverá ser feita com base em critérios estabelecidos pelo grupo, obviamente levando em conta os dados científicos levantados nas etapas anteriores do exercício. Assim, o grupo pode, por exemplo, selecionar as quatro células que contêm os maiores valores conservativos; as células que contêm apenas espécies ameaçadas de extinção; ou ainda realizar as escolhas com base em outros critérios técnicos. O papel do professor é apenas de mediador, levantando as possibilidades existentes e lançando os questionamentos aos alunos para que as decisões dos grupos possam ser bem fundamentadas. 
Assim, com o intuito de promover uma análise crítica das áreas selecionadas por cada um dos grupos, bem como das categorias das áreas protegidas propostas, as seguintes questões norteadoras são apresentadas:

1. As quatro áreas prioritárias para fauna coincidem com as áreas selecionadas para flora?

2. As quatro áreas gerais selecionadas contemplam todas as espécies nativas?

3. Nestas áreas selecionadas existem espécies exóticas?

4. Todas as espécies ameaçadas foram contempladas nas áreas selecionadas?

5. As células com maior riqueza de espécies coincidem com as células de maior valor conservativo?

6. Existem espécies que já estavam preservadas na UC existente (i.e. APA municipal)?

7. As áreas selecionadas estão próximas ou distantes entre si?

8. É possível associar as espécies de maior valor com as áreas de preservação existentes na paisagem?

9. Existiriam outros atributos para a escolha das áreas que poderiam ser levados em conta?

10. Existiria a possibilidade de conciliar a conservação da biodiversidade com o uso sustentável dos recursos naturais da região?

Após a discussão interna de cada grupo, todos devem apresentar seus resultados (i.e. células selecionadas), justificando suas escolhas. Nesse sentido, convém ressaltar que, devido ao reduzido número de células que podem ser selecionadas, dificilmente todas as espécies poderão ser protegidas. Contudo, é importante lembrar que não há uma resposta propriamente correta ou errada para o exercício, pois cada grupo pode se utilizar de critérios próprios, baseados nos conteúdos científicos pesquisados nas etapas anteriores do exercício para subsidiar suas escolhas. A discussão que deve ocorrer, provocada e mediada pelo professor, diz respeito a uma análise crítica das escolhas realizadas, as quais poderão ser distintas dependendo dos critérios e das prioridades de conservação estabelecidos (p. ex. selecionar quatro áreas distantes uma das outras é melhor do que selecionar quatro áreas próximas?; proteger espécies que estão em altos níveis de ameaça (p. ex. "Criticamente em Perigo" - CR) é uma estratégia melhor, ou mais eficiente, do que proteger espécies que estão classificadas como menos ameaçadas?).

Esta etapa final do exercício é considerada como a mais importante, uma vez que é nela que ocorrem as discussões mais profundas, a argumentação técnico-científica sólida dos grupos, o desenvolvimento do senso crítico, além da aplicação concreta dos conceitos previamente trabalhados (i.e. endemismo, distribuição geográfica, espécies ameaçadas, espécies exóticas, áreas prioritárias para conservação, etc.), bem como de outros aspectos relacionadas às dimensões humanas das áreas protegidas e seu entorno.

\section{Possibilidades de adaptação da atividade para outros níveis escolares}

Em virtude da importância e da real necessidade de se iniciar ainda na escola o desenvolvimento de conhecimentos e habilidades relacionadas à sustentabilidade ambiental (Emery et al., 2015; Dionor et al., 2020), é apresentada também uma adaptação deste exercício para os níveis fundamental e médio de ensino. $\mathrm{O}$ desenvolvimento deste conteúdo nestes níveis escolares visa contribuir para a compreensão do importante papel das áreas protegidas para a conservação da biodiversidade, bem como para o desenvolvimento de senso crítico e formação de cidadãos socioambientalmente responsáveis. Neste contexto, é importante ressaltar que a própria BNCC reconhece a educação como forma de afirmar valores e estimular as ações que contribuam para a transformação da sociedade, tornando-a mais humana, justa e voltada para a proteção e preservação da natureza.

Conforme a BNCC, no nono ano do Ensino Fundamental, a "Preservação da Biodiversidade" aparece como um dos objetos de conhecimento propostos, sendo que o aluno deverá conseguir "justificar a importância das UCs para a preservação da biodiversidade e do patrimônio nacional, considerando os diferentes tipos de unidades (parques, reservas e florestas nacionais), as populações humanas e as atividades a elas relacionadas" (Brasil, 2017). Já no Ensino 
Médio, uma das competências específicas de Ciências da Natureza e suas tecnologias a serem desenvolvidas é a investigação de situaçõesproblema e aplicações do conhecimento científico e tecnológico utilizando procedimentos e linguagens próprios das Ciências da Natureza, para propor soluções que considerem demandas locais, regionais e/ou globais (Brasil, 2018).

Diante do exposto acima e dos temas abordados no exercício proposto, essa atividade pode ser adaptada para o nono ano do Ensino Fundamental e para o Ensino Médio, visto o objeto de conhecimento e as habilidades propostas pela BNCC para esses dois níveis de ensino.

\section{Proposta de adaptação para o nono ano do Ensino Fundamental e Ensino Médio}

Com o intuito de readequar a atividade $e$ simplificar as informações a serem analisadas pelos alunos, é sugerida uma redução no número de espécies constantes no exercício. Para tanto, um novo diagrama é apresentado (Figura 3) semelhante à Figura 1, porém com 14 espécies no total, incluindo sete representantes da flora (A-G) e sete correspondentes à fauna $(\mathrm{H}-\mathrm{N})$.

\begin{tabular}{|c|c|c|c|c|c|c|c|c|}
\hline & $\mathbf{a}$ & b & c & d & $\boldsymbol{e}$ & f & $\mathbf{g}$ & $\mathbf{h}$ \\
\hline \multirow{2}{*}{1} & DEF & $\mathrm{CDG}$ & DF & DF & $\mathrm{DF}$ & F & F & $\mathrm{F}$ \\
\hline & I & IK & ILN & IL & ILN & $\mathrm{L}$ & LN & LN \\
\hline \multirow{2}{*}{2} & D & CD & DF & \multirow{2}{*}{ D } & \multirow{2}{*}{$\begin{array}{c}\mathrm{BD} \\
\mathrm{IJ}\end{array}$} & \multirow{2}{*}{$\begin{array}{c}\text { BD } \\
\text { IJ }\end{array}$} & BD & \multirow{2}{*}{ E } \\
\hline & I & HI & HIL & & & & IJN & \\
\hline \multirow{2}{*}{3} & $\mathrm{AD}$ & ACE & E & \multirow{2}{*}{ E } & \multirow{2}{*}{$\begin{array}{c}\mathrm{BE} \\
\mathrm{J}\end{array}$} & \multirow[t]{2}{*}{$\mathrm{BE}$} & BDE & \multirow{2}{*}{$\begin{array}{c}\mathrm{DE} \\
\mathrm{I}\end{array}$} \\
\hline & M & HM & $\mathrm{H}$ & & & & IJN & \\
\hline \multirow{2}{*}{4} & D & CG & FG & A & \multirow{2}{*}{$\begin{array}{c}\mathrm{ABE} \\
\mathrm{JM}\end{array}$} & \multirow{2}{*}{$\begin{array}{c}\mathrm{BE} \\
\mathrm{J}\end{array}$} & BDE & \multirow{2}{*}{$\begin{array}{c}\text { BDE } \\
\text { IJ }\end{array}$} \\
\hline & HI & HK & $\mathrm{KL}$ & MN & & & IJN & \\
\hline \multirow{2}{*}{5} & $\mathrm{DF}$ & $\mathrm{CD}$ & \multirow{2}{*}{ G } & AG & \multirow{2}{*}{ E } & $\mathrm{BE}$ & $\mathrm{BCD}$ & $\mathrm{BCD}$ \\
\hline & IL & I & & $\mathrm{KM}$ & & $J$ & IJN & IJ \\
\hline \multirow{2}{*}{6} & DEF & \multirow{2}{*}{ C } & \multirow{2}{*}{ C } & B & B & $\mathrm{BE}$ & $B C$ & $\mathrm{BC}$ \\
\hline & $\mathrm{L}$ & & & $J$ & $J$ & J & $\mathrm{J}$ & $J$ \\
\hline 7 & DEF & BCDEF & $\mathrm{BCE}$ & $\mathrm{BD}$ & $\mathrm{BDE}$ & $\mathrm{BDE}$ & $\mathrm{BCDE}$ & $\mathrm{BCD}$ \\
\hline & IL & IJL & $\mathrm{J}$ & IJ & IJ & IJ & JLN & IJ \\
\hline 8 & DEF & BDEF & $\mathrm{BDE}$ & $\mathrm{BDE}$ & $\mathrm{BCDEF}$ & BCDEF & $B C D E$ & $\mathrm{BCE}$ \\
\hline & IL & JIL & JI & JI & $\mathrm{JL}$ & $\mathrm{JL}$ & JILN & $\mathrm{J}$ \\
\hline
\end{tabular}

Figura 3 - Diagrama simplificado com a distribuição das espécies da flora (letras A-G) e fauna regional (letras H-N), adaptado para o Ensino Fundamental e Médio. As células em verde claro ("1c" e "1d") representam uma Área de Proteção Ambiental Municipal, onde está localizada a nascente que abastece o rio da região, representado em azul. As espécies destacadas em vermelho são sugeridas como espécies ameaçadas. Fonte: Autores. 
Para a utilização do novo diagrama, o professor deverá elaborar, portanto, uma nova lista de espécies da fauna e flora regional. É interessante que o professor escolha espécies de sua região, intercalando espécies comuns, que sejam bem conhecidas dos alunos, com espécies mais raras e pouco conhecidas. Também é recomendado que sejam selecionadas tanto espécies carismáticas quanto de menor apelo popular, assim como espécies em diferentes graus de ameaça (conforme as listas vermelhas de espécies ameaçadas na região em questão), uma vez que esta diversidade de informações possibilitará um aprofundamento maior nas discussões que se seguirão (Douglas \& Winkel, 2014).

Para estabelecer uma maior conexão entre o conteúdo trabalhado e a realidade socioambiental (Dionor et al., 2020), é sugerido ainda que a proporção de espécies ameaçadas utilizadas seja próxima a $25 \%$ (i.e. de três a quatro espécies do total), visto este ser, aproximadamente, o percentual estimado de espécies ameaçadas de extinção em nível global na atualidade (IUCN, 2020). Assim, para facilitar a organização da atividade pelo professor, são destacadas no diagrama simplificado (Figura 3) duas espécies da fauna $e$ duas da flora que poderiam ser enquadradas em alguma categoria de ameaça.

Para a seleção das espécies em cada região, é interessante contar com a participação dos próprios alunos, como uma forma de introduzir o assunto e valorizar o conhecimento local. Para definição da lista final de espécies da fauna $e$ flora regional, o professor poderá ainda buscar informações nas bases de dados existentes sobre a biodiversidade brasileira. É importante lembrar que, em um segundo momento, o professor deverá acrescentar à lista final informações referentes ao estado de conservação e endemismo de cada espécie, visando facilitar a análise por parte dos alunos. A inclusão destes dados é sugerida uma vez que a busca destas informações pelos próprios alunos poderia requerer algumas habilidades ainda não desenvolvidas e, principalmente, demandaria um tempo excessivo, geralmente não disponível nas aulas de Ciências ou Biologia semanais.

Para a realização do Passo 2, uma vez que as informações sobre o estado de conservação $e$ endemismo serão fornecidas pelo próprio professor, os alunos deverão calcular apenas a informação sobre a raridade de cada uma das espécies presentes na paisagem (i.e. número de células em que a espécie está presente, em relação ao total de células existentes). A partir disto, os alunos deverão fazer a conversão dos resultados encontrados pelos valores da Tabela 3, a fim de obter o valor conservativo de cada uma das espécies presentes na comunidade.

Ainda no Passo 2, é proposto que o professor forneça ou discuta com os alunos informações a respeito de quais são as principais ameaças à biodiversidade existentes em sua região, abordando, se possível, as próprias espécies selecionadas. Estas informações serão importantes para a compreensão da problemática em questão e facilitam a tomada de decisões pelos grupos, bem como as discussões sobre a escolha das áreas prioritárias. Para contextualizar ainda mais o exercício, é sugerido que o professor solicite aos alunos que estes busquem na internet uma foto de cada uma das espécies regionais selecionadas.

O Passo 3 pode ser executado conforme proposto no exercício original, porém levando em consideração o menor número de espécies presentes no diagrama. Em relação ao Passo 4, dependendo do tempo disponível para a atividade ou do grau de conhecimento da turma, o exercício pode ser ainda simplificado excluindo os itens "c" (tipos de UCs) e "d" (definição da zona industrial). Obviamente, esta escolha fica a critério do professor, uma vez que ele mais do que ninguém conhece a sua turma e os conhecimentos que estes possuem, bem como sua capacidade de orientar as discussões dentro do tempo disponível.

Por fim, como sugestões para a apresentação dos resultados da atividade de cada grupo, os mesmos podem:

a) elaborar um grande cartaz (utilizando papel pardo, por exemplo) com o diagrama da área (Figura 3), onde aparecerão as quatro células gerais selecionadas pelo grupo para a conservação;

b) colocar as fotos das espécies que ocorrem dentro das quatro células selecionadas para a UC;

c) escolher um nome para a UC criada;

d) colocar as fotos das espécies que não foram conservadas, ou seja, que não estão nas quatro células selecionadas;

e) escrever outras possibilidades de ações de conservação que poderiam ser realizadas para a preservação das espécies que não estão nas quatro células selecionadas. 
Caso o professor prefira ou tenha a possibilidade de realizar este exercício utilizando computador, a atividade da elaboração do cartaz em papel poderá, por exemplo, ser substituída pela elaboração de um cartaz virtual utilizando ferramentas como Powerpoint, Word, Paint e outras conforme a disponibilidade. O professor também pode solicitar que os alunos representem a área através de desenhos, podendo assim fazer a integração com outras disciplinas como Artes, por exemplo.

Como atividade complementar ainda ao exercício proposto, o professor pode solicitar que os alunos procurem matérias e notícias em jornais locais ou em páginas da internet relacionadas ao tema da extinção de espécies ou às UCs. Além disso, esta proposta de trabalho pode ser implementada de forma interdisciplinar, com a participação de professores de outras disciplinas, cada qual trabalhando conteúdos específicos a partir do tema que está sendo proposto.

\section{Resultados e Discussão}

A atividade de ensino proposta fornece um modelo para a discussão de diversas questões importantes relacionadas às estratégias de conservação e uso sustentável da biodiversidade. Nesse sentido, alguns tópicos que podem ser explorados durante a discussão dos resultados, por exemplo, são os princípios da precaução, complementaridade $e$ insubstituibilidade. $\mathrm{O}$ primeiro preconiza que sempre que houver boas evidências que uma população ou ecossistema é frágil ou ameaçado, deve-se assumir o cenário mais desfavorável, optando pela ação que garanta a melhor proteção nesse cenário (Trajano, 2010). O segundo, também bastante utilizado na priorização de áreas para conservação, baseia-se na ideia de incorporar elementos (e.g. espécies ou ecossistemas) pouco ou não representados nas áreas protegidas já existentes (Margules \& Pressey, 2000; Pinto \& Grelle, 2009). Para contextualizar estes fundamentos, utilizando o princípio da precaução no exercício proposto, uma espécie que aparece na lista como "Dados Deficientes" (DD) deverá ter prioridade de conservação diante de outra espécie classificada como "Menos Preocupante" (LC) (ver Figura 2), recebendo assim uma pontuação maior em relação a este critério (Tabela 3). Neste caso, parte-se do princípio de que a classificação de uma espécie como "Dados Deficientes" reflete simplesmente a falta de informações científicas sobre ela $e$, consequentemente, não se pode descartar completamente a possibilidade de ela estar de alguma forma ameaçada de extinção. Em relação ao princípio da complementaridade, uma espécie que já estivesse preservada na APA municipal da área de estudo, por exemplo, não teria prioridade à outra espécie que ainda não está protegida por nenhuma UC. Por fim, o princípio da insubstituibilidade envolveria aquelas áreaschave ou "áreas insubstituíveis" para os alvos de conservação estabelecidos. Portanto, essas áreas conteriam espécies não representadas em nenhum outro local e se elas forem perdidas dificilmente os objetivos de conservação serão alcançados (Paglia et al., 2004; Padua et al., 2008).

Outro tema certamente bastante interessante de ser abordado na discussão dos resultados envolve um antigo debate no meio científico, especialmente nas décadas de 1970 e 1980, sobre qual seria a melhor opção para a conservação da biodiversidade: a preservação de um único fragmento grande, ou de vários pequenos fragmentos de áreas equivalentes ao fragmento maior (em Inglês, Single Large or Several Small, comumente referido como SLOSS) (Primack \& Rodrigues, 2001; Metzger, 2010). O interessante da discussão desse tema, ainda bastante controverso no meio científico atualmente (MillerRushing et al., 2019), é que, além dos conceitos científicos inerentes (e.g. relação espécie-área, efeito de borda, conectividade) (Figura 4), ele permite explorar a ideia de que existem diferentes possibilidades de manejo, incluindo a discussão dos prós e contras de cada uma delas em diferentes situações (Primack \& Rodrigues, 2001; Donaldson et al., 2017).

A atividade proposta permite ainda incluir outros elementos fundamentais na discussão da conservação da biodiversidade, como as dimensões humanas no interior $e$ entorno das áreas protegidas, incluindo seus aspectos sociais, éticos e culturais (Vivacqua \& Vieira, 2005; Irving \& Matos, 2006; Kopnina, 2016). Nesta discussão, por exemplo, podem ser exploradas diferentes perspectivas de manejo, que incluem desde a exploração dos recursos naturais por populações tradicionais à completa exclusão da presença humana das áreas consideradas prioritárias para conservação (Arruda, 1999; Silveira, 2001; Ferreira, 2004). 


\begin{tabular}{|c|c|c|c|}
\hline Cenário & Menos indicado & Mais indicado & Descrição do melhor cenário \\
\hline 1. & & & Áreas maiores \\
\hline 2. & & & Áreas não fragmentadas \\
\hline 3. & & & $\begin{array}{r}\text { Áreas com formatos } \\
\text { circulares }\end{array}$ \\
\hline 4. & & & $\begin{array}{r}\text { Maior número } \\
\text { de áreas }\end{array}$ \\
\hline 5. & & & Áreas mais próximas \\
\hline 6. & & & $\begin{array}{l}\text { Áreas interligadas por } \\
\text { "pontes" de dispersão }\end{array}$ \\
\hline 7. & & & $\begin{array}{l}\text { Áreas interligadas por } \\
\text { "corredores" }\end{array}$ \\
\hline 8. & & & $\begin{array}{r}\text { Áreas manejadas de } \\
\text { forma conjunta }\end{array}$ \\
\hline
\end{tabular}

Figura 4 - Comparação de alguns cenários envolvendo o delineamento de áreas protegidas. Fonte: Autores, adaptado de Primack \& Rodrigues (2001).

A experiência demonstrou, de fato, que um dos pontos mais positivos e atraentes para os alunos durante a atividade aqui proposta é que ela promove um espaço para avaliação $e$ discussão das informações científicas disponíveis para a tomada de decisão dentro de um contexto interdisciplinar, sendo este um componente central dentro da Biologia da Conservação (Schwartz et al., 2018). Contudo, conforme observado por Noss (1997), os cursos das áreas de ciências ambientais oferecidos pelas universidades, de maneira geral, pouco desenvolvem as habilidades necessárias para a tomada de decisão relacionada a temas socioambientais, os quais muitas vezes podem ser controversos (Lee \& Grace, 2010; Alred \& Dauer, 2020).
Como mencionado anteriormente, estas habilidades deveriam ser exploradas e desenvolvidas inclusive nos anos iniciais de ensino (Emery et al., 2015; Dionor et al., 2020). Para tanto, conforme destacado pelo Comitê de Educação da Sociedade para a Biologia da Conservação, cada professor deve procurar desenvolver tais competências nos seus alunos, explorando os princípios da Biologia da Conservação, de forma a tornar este assunto relevante no contexto do desenvolvimento do conhecimento de seu ambiente local, sem perder de vista a apreciação da condição da biosfera como um todo (Trombulak et al., 2004). Nesse sentido, é interessante mencionar que diversas informações a respeito da distribuição e estado de 
conservação das espécies em todo mundo estão cada vez mais acessíveis (e.g. mapas digitais da distribuição das espécies, tais como Map of LifeJetz et al., 2012), permitindo que as experiências e discussões do exercício aqui apresentado possam ser adaptadas e atualizadas para diferentes regiões $e$ realidades.

Esta proposta de adaptação regional, utilizando informações e espécies mais próximas da realidade dos alunos, é particularmente importante para as turmas do Ensino Fundamental. Conforme destacado por Tozoni-Reis (2006), temas ambientais locais podem ser utilizados como temas geradores de processos educativos ambientais. Para tanto, a referida autora destaca ser fundamental que os temas ambientais sejam contextualizados e que tenham significado social e histórico para os grupos envolvidos, em um processo de aproximação crítica da própria realidade. Partindo do princípio que a extinção de espécies da fauna e da flora regional pode ter um significado concreto para os alunos em diferentes regiões do país e que, certamente, este tema envolve um conteúdo problematizador, a adaptação desse exercício para diferentes realidades locais pode permitir reflexões mais amplas sobre o meio ambiente e a própria relação homem-natureza.

No caso ainda da aplicação do exercício com turmas dos níveis fundamental e médio de ensino, a sugestão de complementação da atividade com a pesquisa de matérias e notícias relacionadas ao tema em questão busca reforçar exatamente a percepção nos alunos da aplicação prática dos conteúdos abordados nos contextos de suas realidades. Essa valorização do conteúdo científico para a aplicação no cotidiano é considerada essencial dentro das perspectivas para o ensino de ciências (Dionor et al., 2020).

Por fim, a adaptação deste exercício prático aos diferentes níveis de ensino visa difundir e fomentar a reflexão não apenas sobre a importância das áreas protegidas, mas também sobre a existência de múltiplas opções válidas para maximizar os esforços relacionados à conservação e uso sustentável dos recursos naturais (e.g. Donaldson et al., 2017). Contudo, é fundamental que as alternativas sejam comparadas $e$ as decisões tomadas com base na mais completa informação científica disponível. Ao mesmo tempo, é fundamental trazer também para a discussão o enorme potencial da contribuição do conhecimento ecológico local para a conservação e manejo da biodiversidade no interior e entorno das áreas protegidas (Arruda, 1999; Padua \& Chiaravalloti, 2012; Gerhardinger et al., 2009; Kellermann et al., 2020).

\section{Conclusão}

O incremento do número e da extensão das UCs no Brasil nas últimas décadas representa, sem dúvida, um avanço para a conservação da biodiversidade brasileira. Contudo, o importante papel dessas áreas protegidas é ainda pouco compreendido por uma grande parcela da população. Nesse sentido, atividades práticas de ensino que estimulem essa reflexão e que façam uso de informações científicas que tenham significado local podem estreitar essa interface, muitas vezes distante, entre ciência, conservação e sociedade.

Portanto, com a atividade proposta, espera-se não apenas facilitar a difusão desta temática em diferentes níveis de ensino, mas criar a oportunidade para o desenvolvimento de habilidades necessárias para analisar $e$ resolver questões relacionadas à conservação da sociobiodiversidade, estimulando também uma maior participação social na gestão das áreas protegidas.

\section{Agradecimentos}

Os autores gostariam de agradecer aos alunos do Mestrado Profissional em Ambiente e Sustentabilidade da Universidade Estadual do Rio Grande do Sul pelas sugestões e discussões realizadas durante a disciplina de Conservação e Manejo da Biodiversidade onde este exercício foi inicialmente desenvolvido. Os autores agradecem também à bióloga e professora Talita da Silva Dewes, pelas importantes contribuições em relação à proposta de adaptação da atividade para os ensinos fundamental e médio, assim como aos revisores, pelas valiosas sugestões para a melhoria da versão final do artigo.

\section{Referências}

Alred AR \& Dauer JM. Understanding factors related to undergraduate student decision-making about a complex socio-scientific issue: mountain lion 
management. Eurasia Journal of Mathematics, Science and Technology Education, 16(2): em 1821, 2020. <https://doi.org/10.29333/ejmste/113757>. Acesso em: 20/08/2020.

Arruda R. "Populações tradicionais" e a proteção dos recursos naturais em unidades de conservação. Ambiente \& Sociedade, 5(2): 79-92, 1999.

Barnosky AD et al. Has the Earth's sixth mass extinction already arrived? Nature, 471(7336): 51-7, 2011.

Brasil. 2002. Decreto $n^{\circ} 4.340$, de 22 de agosto de 2002. Dispõe sobre o Sistema Nacional de Unidades de Conservação da Natureza (SNUC), e dá outras providências. Diário Oficial da União. <http://www. planalto.gov.br/ccivil_03/decreto/2002/D4340.htm > Acesso em: 23/08/2020.

Brasil. 2010. Ministério do Meio Ambiente/Instituto Chico Mendes de Conservação da Biodiversidade, Estratégia Nacional de Comunicação e Educação Ambiental (ENCEA), Brasília.<http://www.icmbio.gov. br/educacaoambiental/images/stories/Politica/politicaencea/encea.pdf $>$. Acesso em 02/02/2021.

Brasil. 2012. Lei n ${ }^{\circ}$ 12.651, de 25 de maio de 2012. Dispõe sobre a proteção da vegetação nativa e dá outras providências. Diário Oficial da União. <http:// www.planalto.gov.br/ccivil_03/_Ato2011-2014/2012/ Lei/L12651.htm>. Acesso èm: 20/08/2020.

Brasil. 2017. Base Nacional Comum Curricular (BNCC). Ensino Fundamental. Brasília: Secretaria da Educação. <http://basenacionalcomum.mec.gov.br/ images/BNCC_EI_EF_110518_versaofinal_site.pdf $>$ Acesso em: 20/08/2020.

Brasil. 2018. Base Nacional Comum Curricular (BNCC). Ensino Médio. Secretaria da Educação. <http://basenacionalcomum.mec.gov.br/images/ historico/BNCC_EnsinoMedio_embaixa_site_110518. pdf $>$. Acesso em: 20/08/2020.

Brooks TM et al. Coverage of the existing global protected area system: is it enough? Bioscience, 54: 1081-1091, 2004.

CDB (Convention on Biological Diversity). 2011. Conference of the Parties Decision X/2: Strategic plan for biodiversity 2011-2020. < www.cbd.int/decision/ cop/?id=12268 $>$. Acesso em: 13/08/2020.

Dionor GA, Conrado DM, Martins L \& Nunes-Neto NF. Avaliando propostas de ensino baseadas em questões sociocientíficas: reflexões e perspectivas para ciências no ensino fundamental. Revista Brasileira de Pesquisa em Educação em Ciências, 20: 429-464, 2020.

Donaldson L, Wilson RJ \& Maclean IMD. Old concepts, new challenges: adapting landscape-scale conservation to the twenty-first century. Biodiversity and Conservation, 26: 527-552, 2017.
Douglas LR \& Winkel G. The flipside of the flagship. Biodiversity and Conservation, 23: 979-997, 2014.

Dudley N, Parrish JD, Redford KH \& Stolton S. The revised IUCN protected area management categories: the debate and ways forward. Oryx, 44: 485-490, 2010.

D’Arrigo RCP, Lorini ML \& Rajão HA. Seleção de áreas para conservação na Mata Atlântica brasileira: revisão dos estudos voltados para priorização espacial. Biodiversidade Brasileira, 10(2): 36-49, 2020.

Eken $\mathrm{G}$ et al. Key biodiversity areas as site conservation targets. Bioscience, 54(12): 1110-1118, 2004.

Emery K, Harlow D, Whitmer A \& Gaines S. Confronting ambiguity in science. The Science Teacher, 82(2): 36-41, 2015.

Fauth G. A ameaçadora volta das extinções em massa. Ciência Hoje, 3(184): 78-79, 2002.

Ferreira LC. Dimensões humanas da biodiversidade: mudanças sociais e conflitos em torno de áreas protegidas no Vale do Ribeira, SP, Brasil. Ambiente \& Sociedade, 7(1): 47-66, 2004.

Franco JLA, Schittini GM \& Braz VS. História da conservação da natureza e das áreas protegidas: panorama geral. Historiæ, Rio Grande, 6(2), 233-270, 2015.

Gerhardinger LC, Godoy EAS \& Jones PJS. Local ecological knowledge and the management of marine protected areas in Brazil. Ocean \& Coastal Management, 52(3-4), 154-165, 2009.

ICMBio (Instituto Chico Mendes de Conservação da Biodiversidade). 2018. Livro Vermelho da Fauna Ameaçada de Extinção no Brasil. Volumes 1-7. Brasília: Ministério do Meio Ambiente. < https://www.icmbio.gov. br/portal/component/content/article/10187>. Acesso em: 13/08/2020

ICMBio (Instituto Chico Mendes de Conservação da Biodiversidade). 2021. Painel dinâmico de informações. Documento não paginado. <http://qvicmbio.gov.br/>. Acesso em 02/02/2021.

IUCN (International Union for Conservation of Nature). 2012. Guidelines for application of IUCN red list criteria at regional and national levels: version 4.0. Gland, Switzerland and Cambridge, UK: IUCN. iii + 41pp. $<$ https://www.iucn.org/content/guidelines-applicationiucn-red-list-criteria-regional-and-national-levelsversion-40>. Acesso em: 20/08/2020.

IUCN (International Union for Conservation of Nature). 2020. The IUCN red list of threatened species. Version 2020-3. <https://www.iucnredlist.org>. Acesso em: $03 / 02 / 2021$. 
Irving MA \& Matos K. Gestão de parques nacionais no Brasil: projetando desafios para a implementação do Plano Nacional Estratégico de Áreas Protegidas. Floresta e Ambiente, 13(2): 89-96, 2006.

Jetz W, McPherson JM \& Guralnick RP. Integrating biodiversity distribution knowledge: toward a global map of life. Trends in Ecology and Evolution, 27: 151159, 2012.

Jones PJS \& de Santo EM. Is the race for remote, very large marine protected areas (VLMPAs) taking us down the wrong track?. Marine Policy, 73: 231-234, 2016.

Kellermann A et al. Conhecimento ecológico local (CEL) na avaliação do estado de conservação de espécies de interesse socioeconômico: integrando saberes na gestão do REVIS Ilha dos Lobos. Biodiversidade Brasileira, 10(3): 41-55, 2020.

Kopnina H. Half the Earth for people (or more)? Addressing ethical questions in conservation. Biological Conservation, 203: 176-85, 2016.

Lee YC \& Grace M. Students' reasoning processes in making decisions about an authentic, local socioscientific issue: bat conservation. Journal of Biological Education, 44(4): 156-165, 2010.

Magris RA \& Pressey RL. Marine protected areas: Just for show?. Science, 360(6390): 2-724, 2018.

Margules CR \& Pressey RL. Systematic conservation planning. Nature, 405: 243-253, 2000.

Margules CR, Pressey RL \& Williams PH. Representing biodiversity: Data and procedures for identifying priority areas for conservation. Biosciense Journal, 27: 309326, 2002.

Martinelli G \& Moraes MA. 2013. Livro Vermelho da Flora do Brasil. 1. ed. - Rio de Janeiro: Andrea Jakobsson: Instituto de Pesquisas Jardim Botânico do Rio de Janeiro. 1100p.

Metzger JP. O Código Florestal tem base científica?. Natureza \& Conservação, 8(1): 92-99, 2010.

Miller-Rushing AJ, Primack RB, Devictor V, Corlett RT, Cumming GS, Loyola R, Maas B \& Pejchar L. How does habitat fragmentation affect biodiversity? A controversial question at the core of conservation biology. Biological Conservation, 232: 271-273, 2019.

MMA (Ministério do Meio Ambiente). Atualização: Portaria MMA n ${ }^{\circ}$ 9, de 23 de janeiro de 2007. < https:// www.icmbio.gov.br/portal/images/stories/portaria mma_092007.pdf > . Acesso em 02/02/2021.

Mittermeier RA, Fonseca GAB, Rylands $A B$ \& Brandon K. Uma breve história da conservação da biodiversidade no Brasil. Megadiversidade, 1(1): 1421, 2005.
Myers N, Mittermeier RA, Mittermeier CG, Fonseca GAB \& Kent J. Biodiversity hotspots for conservation priorities. Nature, 403: 853-858, 2000.

Noss R. The failure of universities to produce conservation biologists. Conservation Biology, 11: 1267-1269, 1997.

Olson D \& Dinerstein E. The Global 200: Priority ecoregions for global conservation. Annals of the Missouri Botanical Garden, 89(2): 199-224, 2002.

Orr DW. 1992. Ecological literacy: education and the transition to a postmodern world. State University of New York Press. 210p.

Padua CV \& Chiaravalloti RM. 2012. Pesquisa e conhecimento na gestão de unidades de conservação, p. 139-153. In: Cases MO (org.). Gestão de Unidades de Conservação: compartilhando uma experiência de capacitação. WWF-Brasil. 396p.

Padua GCC, Pinto MP \& Diniz-Filho JAF. Escolha de áreas prioritárias de conservação de anfíbios anuros do Cerrado através de um modelo de populações centraisperiféricas. Iheringia, Sér. Zool., 98(2): 200-204, 2008.

Paglia APA, Paese L, Bedê M, Fonseca LPP \& Machado RB. 2004. Lacunas de conservação e áreas insubstituíveis para vertebrados ameaçados da Mata Atlântica, p. 39-50. In: Anais do IV Congresso Brasileiro de Unidades de Conservação. Volume II - Seminários. Fundação o Boticário de Proteção à Natureza e Rede Nacional Pró Unidades de Conservação.

Phillips A. The history of the international system of protected area management categories. Parks, 14: 4-14, 2004.

Pimm SL \& Lawton JH. Planning for Biodiversity. Science, 279(5359): 2068-2069, 1998.

Pimm L, Jenkins CN \& Abell R. The biodiversity of species and their rates of extinction, distribution, and protection. Science, 344(6187): 246-752, 2014.

Pinto MP \& Grelle CE. Seleção de reservas: estudos na América do Sul e revisão de conceitos. Oecologia Australis, 13: 498-517, 2009.

Prates APL \& Irving MA. Conservação da biodiversidade e políticas públicas para as áreas protegidas no Brasil: desafios e tendências da origem da $\mathrm{CDB}$ às metas de Aichi. Revista Brasileira de Políticas Públicas, 5(1): 27-57, 2015.

Pressey RL, Cabeza M, Watts ME, Cowling RM \& Wilson KA. Conservation planning in a changing world. Trends in Ecology \& Evolution, 22: 583-592, 2007.

Primack RB \& Rodrigues E. 2001. Biologia da conservação. E. Rodrigues. 327p. 
Rio Grande do Sul. 2013. Portaria da Secretaria de Meio Ambiente $n^{\circ} 79$, de 31 de outubro de 2013. Lista de espécies exóticas invasoras do estado do Rio Grande do Sul, 2013. <https://www.sema.rs.gov.br/ legislacao-relacionada-5ea3356e6b14c>. Acesso em: 21/08/2020.

Rio Grande do Sul. 2014a. Decreto Estadual n 51.797, de 8 de dezembro de 2014. Lista das espécies da fauna nativa ameaçadas de extinção. < http://www.fzb.rs.gov. br/upload/20140919104139decreto51797.pdf >. Acesso em: 20/08/2020.

Rio Grande do Sul. 2014b. Decreto Estadual n 52.109, de 19 de dezembro de 2014. Lista das espécies da flora nativa ameaçadas de extinção. < http://www.fzb.rs.gov. br/upload/20141222103647doe.pdf $>$. Acesso em: 20/08/2020.

Rodrigues ASL et al. Effectiveness of the global protected area network in representing species diversity. Nature, 428: 640-643, 2004.

Rodrigues ASL, Pilgrim JD, Lamoreux JL, Hoffmann M $\&$ Brooks TM. The value of the Red List for conservation. Trends in Ecology \& Evolution, 21: 71-76, 2006.

Santos WLP \& Mortimer EF. Tomada de decisão para ação social responsável no Ensino de Ciências. Ciência \& Educação, 7(1): 95-111, 2001.

Silva AL. A meta 11 de Aichi e as áreas marinhas protegidas em grande escala: proteção ambiental ou oportunismo político?. Revista de Direito Internacional, 16(2): 39-53, 2019.

Silveira PCB. Parks in peril: people, politics and protected areas. Ambiente \& Sociedade, 9: 157-162, 2001.

Schwartz MW et al. Decision support frameworks and tools for conservation. Conservation Letters, 11: 1-12, 2018.
Tali BA, Khuroo AA, Nawchoo IA \& Ganie AH. Prioritizing conservation of medicinal flora in the himalayan biodiversity hotspot: an integrated ecological and socioeconomic approach. Environmental Conservation, 46(2): 147-154, 2019.

Tozoni-Reis MFC. Temas ambientais como "temas geradores": contribuições para uma metodologia educativa ambiental crítica, transformadora $e$ emancipatória. Educar em Revista, 27: 93-110, 2006.

Trajano E. Políticas de conservação e critérios ambientais: princípios, conceitos e protocolos. Estudos Avançados, 24(68): 135-146, 2010.

Trombulak SC et al. Principles of conservation biology: recommended guidelines for conservation literacy from the education committee of the Society for Conservation Biology. Conservation Biology, 18: 1180-1190, 2004.

Venter O, Fuller RA, Segan DB, Carwardine J \& Brooks T. Targeting global protected area expansion for imperiled biodiversity. PLOS Biology 12(6): e1001891j, 2014. <https://doi.org/10.1371/journal.pbio.1001891>. Acesso em: 20/08/2020.

Visconti $P$ et al. Protected area targets post-2020. Science, 364: 239-241, 2019.

Vivacqua M \& Vieira PHF. Conflitos socioambientais em Unidades de Conservação. Política e Sociedade, 4(7): 139-162, 2005.

Watson JEM, Dudley N, Segan D \& Hockings M. The performance and potential of protected areas. Nature, 515: 67-73, 2014.

Ziller SR \& Zalba S. Propostas de ação para prevenção e controle de espécies exóticas invasoras. Natureza \& Conservação, 5(2): 8-15, 2007.

Biodiversidade Brasileira - BioBrasil.

Fluxo Contínuo

n. 3, 2021

http://www.icmbio.gov.br/revistaeletronica/index.php/BioBR

Biodiversidade Brasileira é uma publicação eletrônica científica do Instituto Chico Mendes de

Conservação da Biodiversidade (ICMBio) que tem como objetivo fomentar a discussão e a disseminação de experiências em conservação e manejo, com foco em unidades de conservação e espécies ameaçadas.

ISSN: 2236-2886 